\title{
Novel compound heterozygous variant of $B S C L 2$ identified by whole exome sequencing and multiplex ligation-dependent probe amplification in an infant with congenital generalized lipodystrophy
}

\author{
BOBO XIE ${ }^{1}$, XIN FAN $^{1}$, YAQIN LEI ${ }^{1}$, SHANG $^{1}{ }^{1}$, QI YANG $^{1}$, JIN WANG $^{1}$, \\ ZAILONG QIN $^{1}$, FEI SHEN ${ }^{1}$, JINGSI LUO ${ }^{1}$ and YIPING SHEN ${ }^{1,2}$ \\ ${ }^{1}$ Department of Genetic and Metabolic Central Laboratory, Guangxi Maternal and Child Health Hospital, \\ Nanning, Guangxi 530023, P.R. China; ${ }^{2}$ Division of Genetics and Genomics, Department of Neurology, \\ Boston Children's Hospital, Harvard Medical School, Boston, MA 02115, USA
}

Received September 18, 2019; Accepted March 6, 2020

DOI: $10.3892 / \mathrm{mmr} .2020 .11036$

\begin{abstract}
Congenital generalized lipodystrophy (CGL) is a clinically and genetically heterogeneous condition with autosomal recessive inheritance. CGL is classified into four subtypes on the basis of causative genes. This study reported on a 2-month-old male infant diagnosed with CGL with generalized lipoatrophy and skin hyperpigmentation. Whole exome sequencing (WES) identified a heterozygous small insertion (c.545_546insCCG) in Berardinelli-Seip congenital lipodystrophy 2 (BSCL2) that was inherited from the infant's mother. Copy number variation analysis using exome data suggested a heterozygous deletion involving exon 3 that was inherited from the infant's father. This finding was confirmed by multiplex ligation-dependent probe amplification test. Gap-PCR revealed breakpoints and confirmed a 1274 bp heterozygous deletion encompassing exon 3 of BSCL2 (c.213-1081_c.294+111). This deletion is different from the founder $3.3 \mathrm{~kb}$ deletion involving
\end{abstract}

Correspondence to: Dr Jingsi Luo, Department of Genetic and Metabolic Central Laboratory, Guangxi Maternal and Child Health Hospital, 59 Xiangzhu Road, Nanning, Guangxi 530023, P.R. China E-mail: ljs0815freedom@163.com

Professor Yiping Shen, Division of Genetics and Genomics, Department of Neurology, Boston Children's Hospital, Harvard Medical School, 300 Longwood Avenue, Boston, MA 02115, USA E-mail: shen.shenyiping@gmail.com

Abbreviations: BSCL2, Berardinelli-Seip congenital lipodystrophy 2; WES, Whole exome sequencing; MLPA, multiplex ligation-dependent probe amplification; CNV, copy number variation

Key words: congenital generalized lipodystrophy, BSCL2, whole exome sequencing, multiplex ligation-dependent probe amplification, deletion exon 3 of BSCL2 in the Peruvian population. An 11-bp microhomology at the breakpoints may mediate the deletion, and its presence indicates the independent origins of the exon 3 deletion between Chinese and Peruvian populations. The present results expanded the mutational spectrum of the BSCL2 gene in the Chinese population and suggested that introns 2 and 3 of BSCL2 are prone to recombination. Thus, exon 3 deletion should be considered for patients with CGL2 when only one BSCL2 variant is detected through WES.

\section{Introduction}

Congenital generalized lipodystrophy (CGL) is a group of rare inherited lipodystrophies characterized by lipoatrophy at birth or in early life $(1,2)$. The clinical presentation of CGL includes acromegaloid features, hypertriglyceridemia and complications related to insulin resistance (1). Four subtypes of CGL have been reported, all of which are autosomal recessive disorders (3). CGL1 (MIM \#608594) and CGL2 (MIM \#269700) result from pathogenic variants in the 1-acylglycerol-3-phosphate $O$-Acyltransferase 2 gene and Berardinelli-Seip congenital lipodystrophy 2 (BSCL2) gene, respectively (3). These are the major forms of CGL, accounting for $95 \%$ of reported CGL cases (4). CGL4 (MIM \#613327), which accounts for $\sim 5 \%$ of cases, is caused by pathogenic variants in the polymerase 1 and transcript release factor $(P T R F)$ gene $(3,4)$. CGL3 (MIM \#612526), which is due to pathogenic variants in the caveolin $1(C A V I)$ gene, has been rarely reported (5). Given the genetic heterogeneity of this condition, exome sequencing has been the method of choice for the molecular diagnosis of CGL. Although whole exome sequencing (WES) can effectively detect single-nucleotide variants (SNVs) and small insertions/deletions, its use to detect copy number variations (CNVs) remains challenging (6).

A founder deletion encompassing exon 3 of BSCL2 had been reported in Peruvian patients (7). Other than that, exonic deletions in BSCL2 are rare. In the present study a small heterozygous insertion and a large heterozygous deletion 
similar to the Peruvian deletion in BSCL2 were detected in the patient. It was demonstrated that the deletions differ in size and breakpoints, indicating that they arose independently.

\section{Materials and methods}

Case presentation. A 2-month-old male infant was referred to the Pediatric Endocrine Department of Guangxi Maternal and Child Health Hospital for skin hyperpigmentation and developmental delay on April 20 ${ }^{\text {th }}$, 2018. Written informed consent for the infant and his parents was obtained from the infant's parents. The present study was approved by the Medical Ethics Committee of Guangxi Maternal and Child Health Hospital. Abdominal ultrasound was applied to assess the presence of hepatomegaly, and cardiac function was assessed by echocardiography and electrocardiography. Blood glucose, blood lipid and C-peptide levels were determined as previously reported (8). Serum insulin and sex hormone levels were measured by electrochemical luminescence immunoassay (Cobas E601; Roche Diagnostics).

WES and CNV analysis. Genomic DNA was extracted from $2 \mathrm{ml}$ peripheral blood of the infant and his parents in accordance with the standard method of the Lab-Aid ${ }^{\circledR}$ DNA extraction kit (Xiamen Zeesan Biotech Co, Ltd.). Human exome sequencing libraries were constructed using Agilent SureSelect Human All Exon V5 kit (Agilent Technologies, Inc.), and generated amplicons were sequenced with Illumina HiSeq 2500 system (Illumina, Inc.). After sequencing, reads were aligned to an indexed human reference genome (GRCh37/hg19) with Burrows-Wheeler transformation 0.7.15-r1140 (9). Duplicate reads were removed using Picard v.1.85 (http://picard. sourceforge.net) before further processing. Base recalibration and variant calling were performed using the Genome Analysis Toolkit v.2.3-4Lite (10). Finally, identified variants were saved in variant call format. In addition, in the present study there was an attempt to reveal CNVs with a read depth-based $\mathrm{CNV}$ detection algorithm. Translational Genomics Expert (LifeMap Sciences, Inc.) was used for variant prioritization. Integrative Genomics Viewer 2.4.15 (IGV 2.4.15) was used to visualize WES data and assess the coverage of the exons (http://software.broadinstitute. org/software/igv/). Variants detected in the infant, but absent in the ClinVar (www.ncbi.nlm.nih.gov/clinvar/), Human Gene Mutation Database (www.hgmd.cf.ac.uk/ac/), Single Nucleotide Polymorphism Database (dbSNP; www.ncbi.nlm. nih.gov/SNP), 1000 Genomes Project database (http://www. internationalgenome.org/1000-genomes-browsers/), Ensembl (http://grch37.ensembl.org/index.html) and Genome Aggregation Database 2.1 (gnomAD 2.1; https:/gnomad. broadinstitute.org) were considered novel variants (11).

Sanger sequencing and multiplex ligation-dependent probe amplification (MLPA). Sanger sequencing was performed to confirm the variant identified by WES. Specific primers for amplification and sequencing were designed in Primer3web version 4.1.0 (http://primer3.ut.ee/). The primer sequences of BSCL2 exon 6 were as follows: forward, 5'-CTACTCAGGG GTGGTTGAGG-3' and reverse, 5'-CCCCACTTCCAGTCTCT CAG-3'. A pair of primers encompassing exon 3 was used to determine the breakpoints (BSCL2_BP3-forward: 5'-TCCCTT AAGTCCCTGGTCCA-3'; BSCL2_BP3-reverse: 5'-ACATTT CTAACACCTGCTGCAG-3'). The PCR product was sequenced with the 3730xl Genetic Analyzer (Applied Biosystems; Thermo Fisher Scientific, Inc.). Sequence data were aligned with the consensus coding sequence [human genome assembly 37 in nucleotide Basic Local Alignment Search Tool (BLAST; http://blast.ncbi.nlm.nih.gov/blast/)]. Mutation Surveyor software (https://softgenetics.com/mutationSurveyor.php; version 4.0.4) was used in visualizing the sequences.

To confirm the suspected exon 3 deletion in BSCL2, MLPA probes were designed and validated following a previous method (12) and the protocol from MRC-Holland BV (https://support.mlpa.com/downloads/files/designing-syntheti c-mlpa-probes). A total of 11 pairs of MLPA probes were designed for BSCL2 (Table SI). These probes were dissolved and diluted in TE buffer, then added to the P200 reference probe mix, which was purchased from MRC-Holland BV and included reference probes and control fragments. The MLPA experiment was performed in accordance with the manufacturers' protocols, with MLPA reagents provided by MRC-Holland BV. Capillary electrophoresis was performed with the 3130 Genetic Analyzer (Applied Biosystems; Thermo Fisher Scientific, Inc.), and data were analyzed with Coffalyser (https://www.mrcholland.com/).

\section{Results}

Clinical evaluation. The infant was the second child born to nonconsanguineous healthy parents. He was a full-term baby with normal delivery, and he had a healthy sister. His weight was 2,500 $\mathrm{g}(-1 \mathrm{SD})$ at birth and 3,900 $\mathrm{g} \mathrm{(-3} \mathrm{SD,} \mathrm{which}$ indicates that the weight of the infant was lower than healthy infants) at 2 months. He had a thin appearance and advanced bone age ( 3 months). Generalized lipoatrophy and skin hyperpigmentation were observed. Moreover, he had a triangular face, marked umbilical prominence, a long and large penis, and a swollen right scrotum (Fig. 1). Echocardiography and abdominal ultrasound indicated atrial septal defect and hepatomegaly, respectively, and no splenomegaly. Liver function tests were normal. Other abnormal biochemical examinations were as follows: Insulin and C-peptide levels were elevated [956.6 pmol/1, normal range (NR): 17.8-173 pmol/1 and $>21.85 \mathrm{ng} / \mathrm{ml}$, NR: 1.1-4.4 $\mathrm{ng} / \mathrm{ml}$, respectively], which indicated insulin resistance. The triglyceride level was increased (26.63 mmol/1, NR: 0.56-1.70 $\mathrm{mmol} / \mathrm{l})$, whereas the levels of high-density lipoprotein cholesterol $(0.52 \mathrm{mmol} / \mathrm{l}$, NR: $1.16-1.55 \mathrm{mmol} / \mathrm{l})$, low-density lipoprotein cholesterol (2.36 mmol/1, NR: 2.70-3.13 mmol/l), apolipoprotein A1 (0.52 g/l, NR: 1.20-1.60 g/l), and apolipoprotein B (0.67 g/l, NR: 0.80-1.05 g/l) were all decreased, indicating hypertriglyceridemia. Sex hormone levels were decreased also (testosterone: $0.85 \mathrm{ng} / \mathrm{ml}, \mathrm{NR}$ : $1.75-7.81 \mathrm{ng} / \mathrm{ml}$; estradiol: $5.00 \mathrm{pg} / \mathrm{ml}, \mathrm{NR}: 20.00-75.00 \mathrm{pg} / \mathrm{ml}$ ).

Genetic findings. WES revealed a c.545_546insCCG heterozygous variant in exon 6 of the BSCL2 gene (GenBank accession no. NM_032667.6). This variant was confirmed by Sanger sequencing (Fig. 2B). It led to a disruptive inframe 

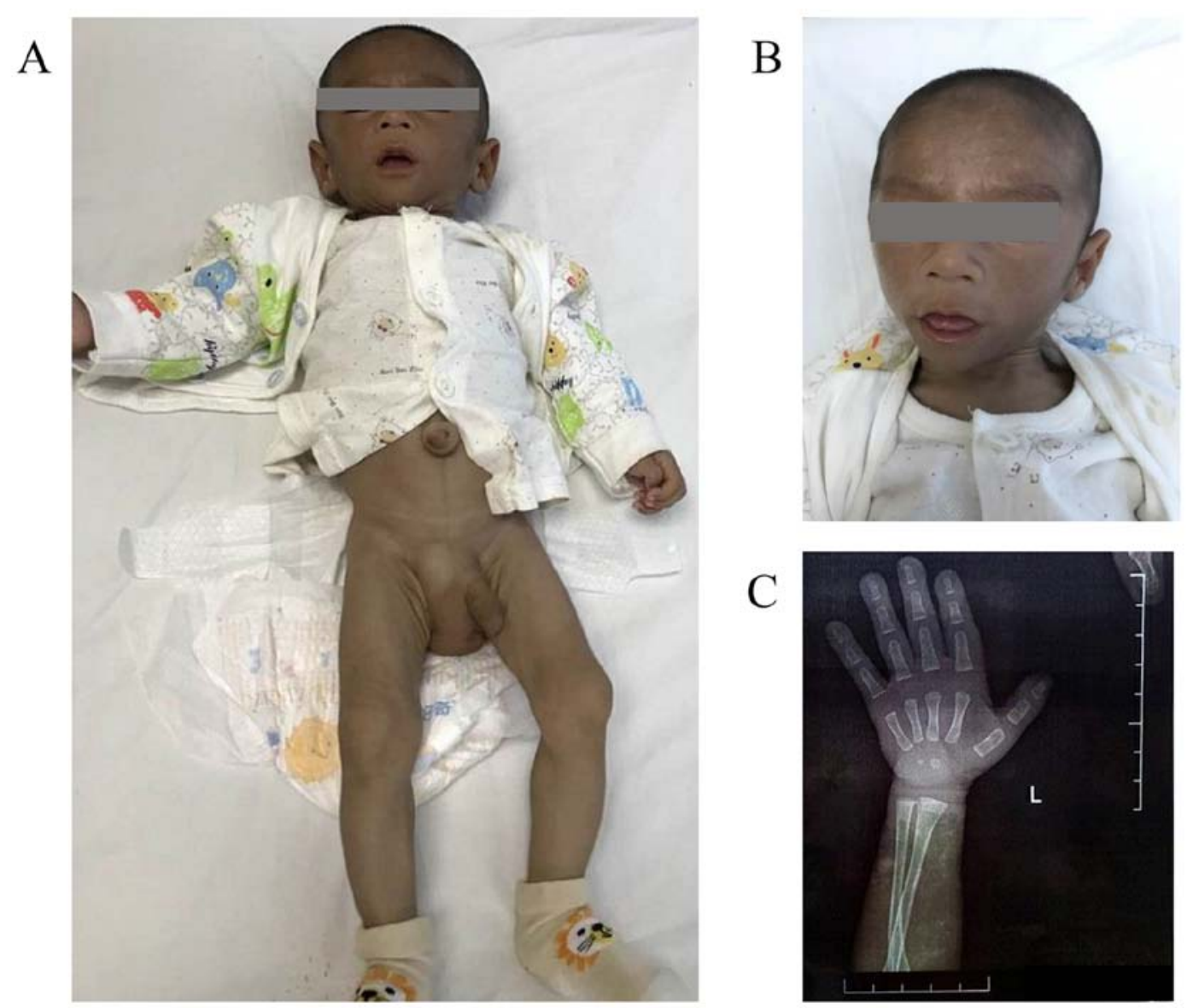

Figure 1. Clinical features of the infant with congenital generalized lipodystrophy. (A and B) Images showing pigmentation of skin, triangular face, marked umbilical prominence, long and large penis and swelling of the right scrotum. (C) X-ray revealing advanced bone age.

indel at position 182 (p.Glu182delinsAspArg). The variant was inherited from the mother. This variant has been documented in the dbSNP database (rs747297291) and at an extremely low frequency in the gnomAD database. It has been detected in trans with a pathogenic variant in a Chinese patient with CGL (13), indicating that it is a recurrent pathogenic variant in the Chinese population.

There were no additional SNVs detected in BSCL2 or any other rare SNVs that could explain the phenotypes. CNV assessment by the visual review of BSCL2 coverage using the IGV browser suggested a heterozygous deletion at 11q12.3 $(62,469,824-62,470,137)$ involving exon 3 of BSCL2 (Fig. 2A). To confirm this heterozygous deletion, MLPA probes covering exon 3 of $B S C L 2$ and other control regions were designed. The copy number ratios of the $B S C L 2$ exon 3 of the infant, his father and his mother were $0.65,0.62$ and 1.01 , respectively (Fig. 2C), indicating the presence of an Ex3del in the infant and his father. The Ex3del was a null variant and absent in the dbSNP, the 1000 Genomes Project database, Ensembl, gnomAD and local normal controls. Notably, a similar exon 3 deletion has been reported as a founder mutation in Peruvian patients with CGL (7).

Gap-PCR was performed to further confirm and map the exact breakpoints of the exon 3 deletion. As revealed in Fig. 3A, a 483-bp amplicon that represented the deleted allele was present in the proband and in the father but not in the mother. Sequencing this amplicon confirmed the deletion and revealed the breakpoints of a 1,274 bp deletion (chr11:62,469,829-62,471,102; c.213-1081_c.294+111), including complete exon 3 and part of introns 2 and 3 (Fig. 3B and C). An 11-bp microhomology was identified at the breakpoints. Thus, this deletion is different from the founder deletion variant detected in Peruvian patients with CGL (7). The deletion would lead to a frameshift and premature termination (p.Thr72Cysfs*2) that is predicted to result in nonsense-mediated mRNA decay.

The maternally inherited variant c.545_546insCCG is rare in the general population (PM2) $(13,14)$. It has been reported in trans with a pathogenic variant at least twice (PM3_Strong) and shortens the protein product (PM4) (13). The patient's phenotype is consistent and specific with CGL (PP4). This variant is classified as likely pathogenic on the basis of ACMG/AMP guidelines for variant classification (15). In the present study, it was speculated that Ex3del was a loss-of-function variant (PVS1) that was absent from the general population (PM2), and PP4 was also applicable; thus, it can be classified as pathogenic.

\section{Discussion}

The main clinical feature of CGL is adipose tissue loss resulting in abnormal carbohydrate and lipid metabolism. The accumulation of ectopic fat can lead to hepatomegaly, dyslipidemia, insulin resistance and diabetes (16). The patient reported 
A

\begin{tabular}{l|l|l|l|l|}
\hline &
\end{tabular}

B

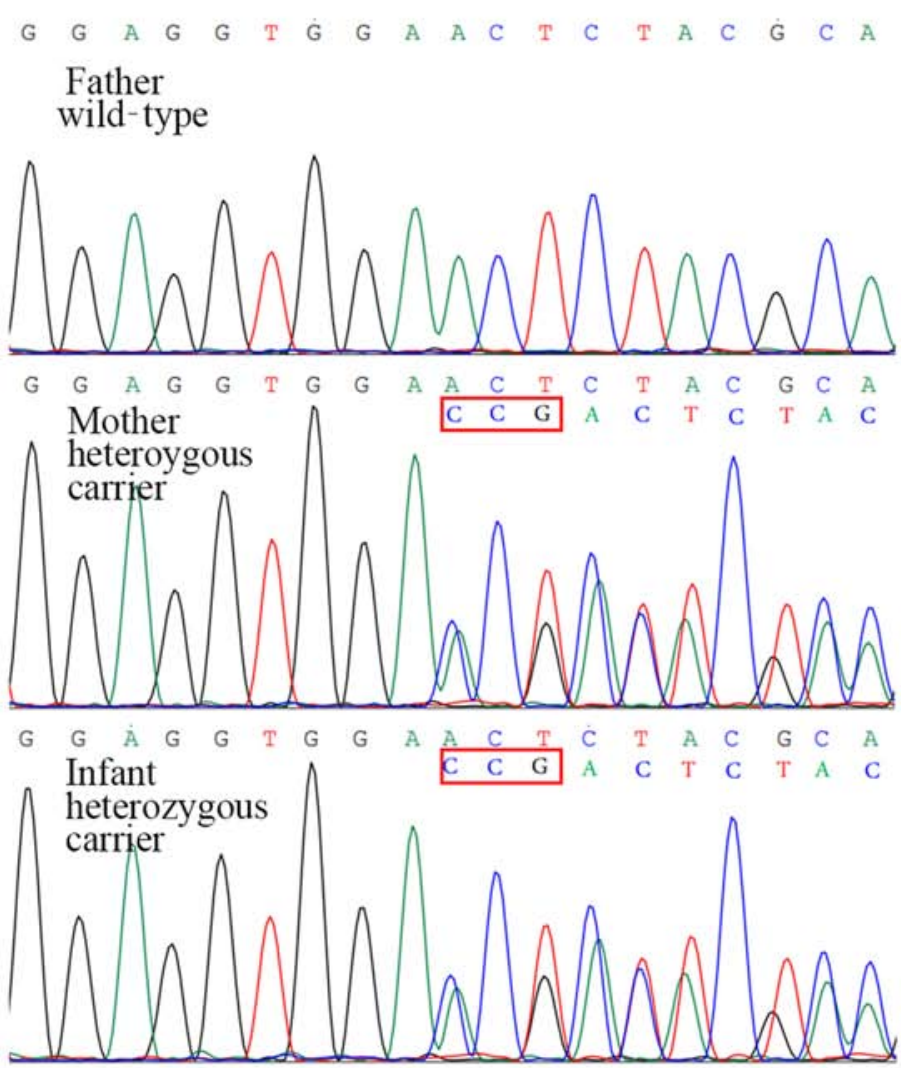

BSCL2 c.545_546insCCG
C

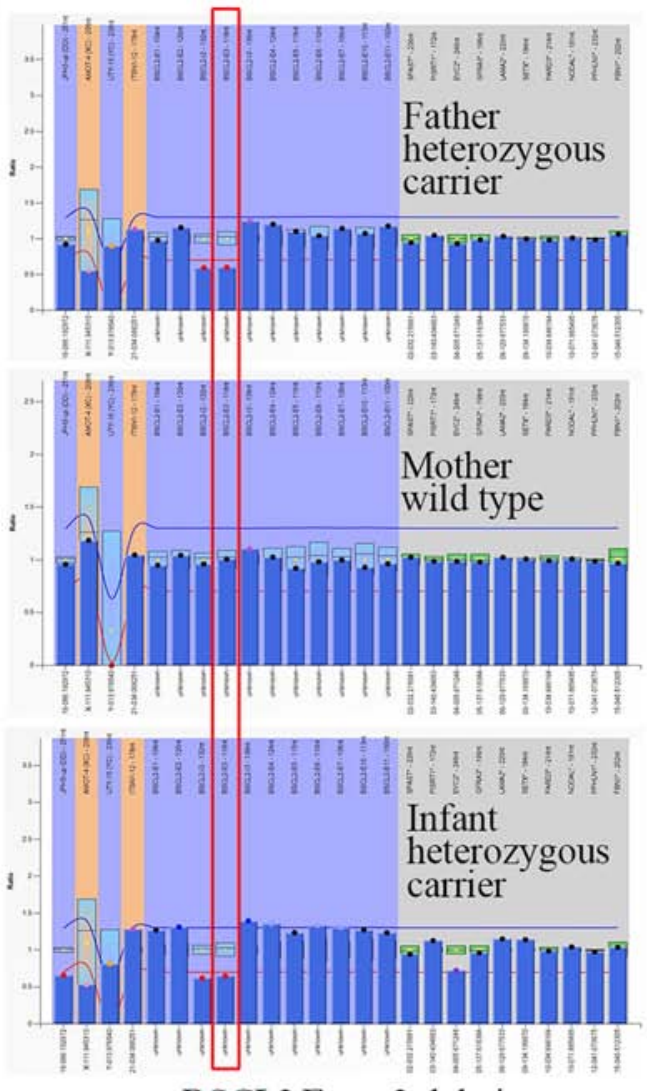

BSCL2 Exon 3 deletion

Figure 2. Genetic findings in the family. (A) IGV image of partial sequences in BSCL2 gene in the infant and controls. (B) Sequencing chromatograms of the infant and the parents of the infant for the variant of c.545_546insCCG. The red frame indicates the inserted sequence 'CCG'. (C) Multiplex ligation-dependent probe amplification targeted to $B S C L 2$ gene that revealed the heterozygous deletion intron 2 and exon 3 for the infant and his father. IGV, Integrative Genomics Viewer; BSCL2, Berardinelli-Seip congenital lipodystrophy 2.

in this study presented with facial abnormalities, marasmus, a macropenis, hepatomegaly and hypertriglyceridemia, which were in accordance with the diagnosis of CGL.

Given that CGL is a genetically heterogeneous disorder, identifying the causative mutation for patients by Sanger sequencing is challenging. In the present study, WES was demonstrated to be useful in detecting small insertions, as well as CNVs that cannot be identified by Sanger sequencing.

CNV detection may increase the diagnostic yield of WES (17), an important issue in genetic diagnosis. There are several algorithms to call CNV from WES data, but no one can detect all CNVs in different size ranges (18-20). A read-depth based algorithm was developed to predict CNVs, which are confirmed in the IGV browser. Although it cannot identify the precise breakpoint of the deletion, the $\mathrm{CNV}$-calling program was successful at predicting heterozygous deletions, including BSCL2 exon 3.

CNV analysis based on exome data is useful but must be confirmed with orthogonal methods. CNV validation methods include chromosomal microarray analysis, reverse transcription-quantitative PCR and MLPA (21). In the present study, MLPA probes were designed to confirm the Ex3del 

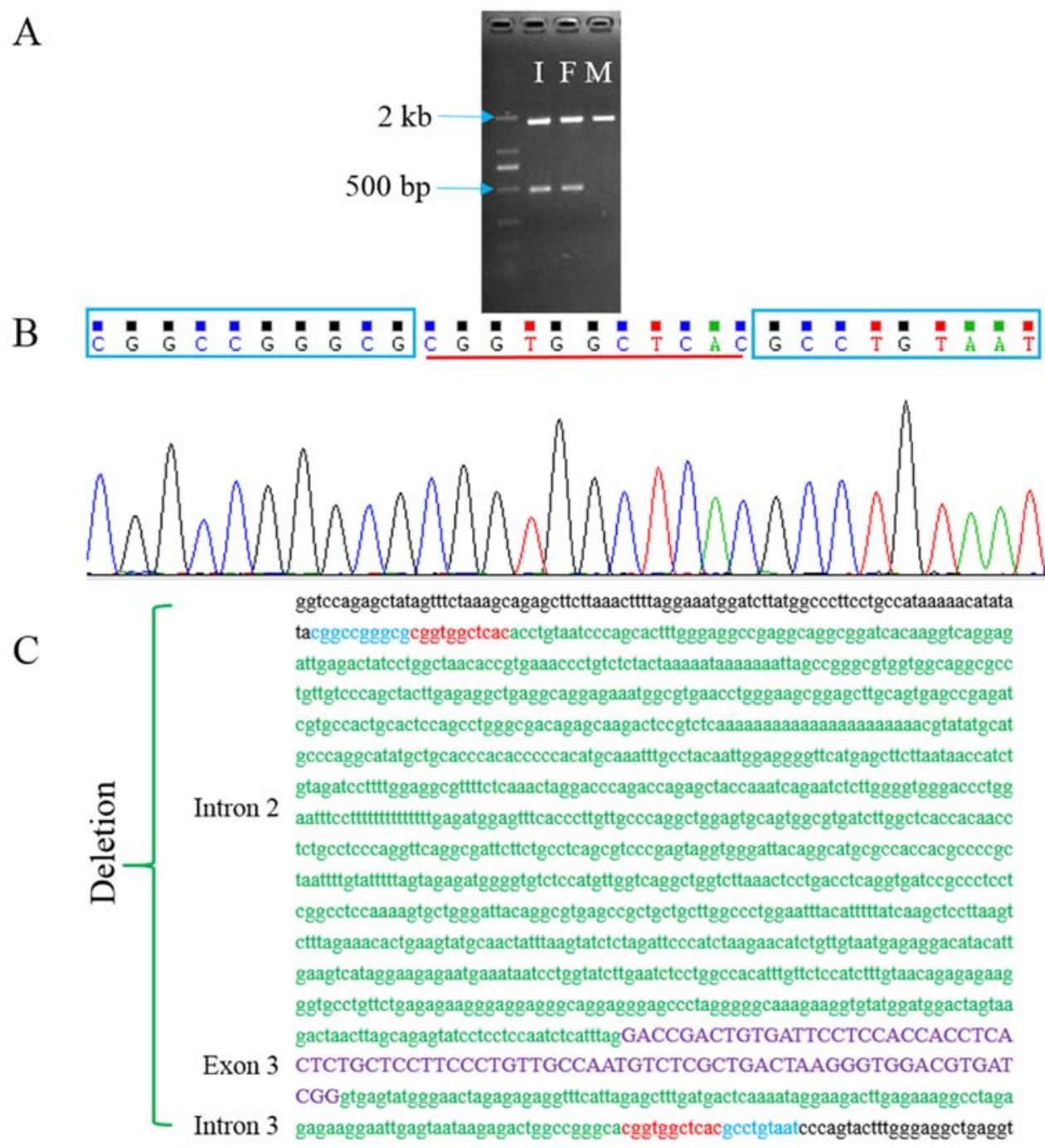

Figure 3. Breakpoint analysis of the BSCL2 Ex3del. (A) Gap-PCR results in the infant (I), father (F) and mother (M), using BP3-foward and BP3-reverse primers. (B) Partial chromatogram of the breakpoint sequence in the DNA of the infant. The blue frames and red underlines indicate the sequences near upstream and downstream breakpoints and the homologous sequences, respectively. (C) Illustration of the deleted sequence among introns 2 and 3. Green lowercase letters indicate the deleted nucleotides in introns 2 and 3. Purple uppercase letters are the deleted exon 3. Red lowercase letters are the homologous sequences of 'CGGTGGCTCAC'. Sequences near upstream and downstream breakpoints are presented as blue lowercase letters. BSCL2, Berardinelli-Seip congenital lipodystrophy 2 .

by designing specific MLPA probes for the region. Further breakpoint analysis by Sanger sequencing revealed a 1,274 bp deletion (chr11:62,469,829-62,471,102), including the complete exon 3 and part of introns 2 and 3. A BLAST search of the flanking junction sequences of the breakpoints was performed, and it was detected that the breakpoint in intron 2 is part of the 303-bp repeat AluY (chr11:62,470,820-62,471,122), and the breakpoint in intron 3 is part of the 293-bp repeat AluSp (chr11:62,469,545-62,469,837). The overall similarity of AluSp and AluY is 80\%, and both of the Alu elements are at the same orientation at the two sites. In particular, an overlapping sequence CGGTGGCTCAC is present at chr11:62,471,103-62,471,114 and chr11:62,469,817-62,469,828. The overlapping 11-bp sequences may mediate a crossover event that lead to deletion. To date, nearly one-quarter of the mutations in BSCL2 reported in patients with type 2 CGL are missense mutations; the others are null mutations, including nonsense, splicing, small indels, small insertions and deletions $(3,22)$. Large deletions have rarely been reported, except for the exon 3 deletion. A 3.3-kb homozygous deletion of exon 3 was reported as a founder mutation in Peruvian population, which appeared to be mediated by recombination between AluSq2 and AluSx3 in introns 2 and 3 (7). The size of the deletions and the breakpoints of Ex3del are different in the Chinese and Peruvian CGL patients, indicating they are independent events (7). However, as aforementioned, both events may be mediated by a similar mechanism and result in the same consequence. Thus, this independent deletion is suggestive of a recurrent event involving exon 3 . Therefore, the exon 3 deletion should be considered for patients with CGL2 when only one BSCL2 variant was detected by exome sequencing.

Glu182, a site that is highly conserved among numerous species, is localized in the conserved loop of the ER lumen $(13,23)$. The variant of c.545_546insCCG resulted in a disruptive inframe indel at position 182 (p.Glu182delinsAspArg), which may cause the misfolding of the secondary structure of the seipin protein due to the insertion of the basic amino acid arginine and could 
destabilize the protein structural domain. Given that this variant has only been reported in Chinese patients with CGL $(13,14)$, it is a recurrent pathogenic variant in the Chinese population.

In conclusion, a novel compound heterozygous variant (p.Glu182delinsAspArg/Ex3del) in the BSCL2 gene was identified in a Chinese infant with CGL by WES. The present study revealed an independent recombination event in introns 2 and 3 that resulted in exon 3 deletion (c.213-1081_c.294+111). This finding expanded the mutational spectrum of the BSCL2 gene while providing further evidence of exonic deletion in the BSCL2 gene in patients with CGL.

\section{Acknowledgements}

Not applicable.

\section{Funding}

The present study was supported by the Major Research Plan of the Provincial Science and Technology Foundation of Guangxi (grant no. AB16380214); the National key research and development program (grant no. 2018YFC1002501); the 'Eastern Scholar' Fund; the 'Guangxi Bagui Scholar' fund; the Natural Science Foundation of China (grant no. 81873633) and GeneScience Pharmaceuticals Co., Ltd. (grant no. xixueyanjiubu-20150808-1). The funding body played no direct role in the design of the study, or in the collection, analysis and interpretation of data, or in the writing of the manuscript.

\section{Availability of data and materials}

The data used and analyzed during the present study are available from the corresponding author or first author on reasonable request.

\section{Authors' contributions}

YS and BX designed the study and experiments. JL and XF analyzed the clinical phenotype and followed up the infant. ZQ and FS exacted DNA and performed the pathogenicity evaluation of gene variation. SY and JW worked on the experiments and bioinformatics analysis of WES. QY performed the Sanger sequencing experiments. YL performed the MLPA experiments and collected the data. BX designed the probes for MLPA and wrote the manuscript. All authors read and approved the final manuscript.

\section{Ethics approval and consent to participate}

The present study was approved by Guangxi Maternal and Child Health Hospital Medical Ethics Committee [approval no. (2018) Lun Han Shen (5-5)] and written informed consent for the infant and his parents was obtained from the infant's parents.

\section{Patient consent for publication}

Written informed consent for the infant was obtained from his parents for the publication of this report, including images and data.

\section{Competing interests}

The authors declare that they have no competing interests.

\section{References}

1. Dantas de Medeiros JL, Carneiro Bezerra B, Brito de Araújo TA, Craveiro Sarmento AS, de Azevedo Medeiros LB, Peroni Gualdi L, Luna Cruz MDS, Xavier Nobre TT, Gomes Lima J and Araújo de Melo Campos JT: Impairment of respiratory muscle strength in Berardinelli-Seip congenital lipodystrophy subjects. Respir Res 19: 173, 2018.

2. Cheema HA, Malik HS, Waheed N, Mushtaq I, Fayyaz Z and Anjum MN: Berardinelli-Seip Congenital Generalised Lipodystrophy. J Coll Physicians Surg Pak 28: 406-408, 2018.

3. Patni N and Garg A: Congenital generalized lipodystrophies new insights into metabolic dysfunction. Nat Rev Endocrinol 11: 522-534, 2015.

4. Boutet E, El Mourabit H, Prot M, Nemani M, Khallouf E, Colard O, Maurice M, Durand-Schneider AM, Chrétien Y, Grès $\mathrm{S}$, et al: Seipin deficiency alters fatty acid Delta9 desaturation and lipid droplet formation in Berardinelli-Seip congenital lipodystrophy. Biochimie 91: 796-803, 2009.

5. Kim CA, Delépine M, Boutet E, El Mourabit H, Le Lay S, Meier M, Nemani M, Bridel E, Leite CC, Bertola DR, et al: Association of a homozygous nonsense caveolin-1 mutation with Berardinelli-Seip congenital lipodystrophy. J Clin Endocrinol Metab 93: 1129-1134, 2008

6. Yao R, Zhang C, Yu T, Li N, Hu X, Wang X, Wang J and Shen Y: Evaluation of three read-depth based CNV detection tools using whole-exome sequencing data. Mol Cytogenet 10: 30, 2017.

7. Purizaca-Rosillo N, Mori T, Benites-Cóndor Y, Hisama FM, Martin GM and Oshima J: High incidence of BSCL2 intragenic recombinational mutation in Peruvian type 2 Berardinelli-Seip syndrome. Am J Med Genet A 173: 471-478, 2017.

8. Lima JG, Nobrega LH, de Lima NN, do Nascimento Santos MG, Baracho MF and Jeronimo SM: Clinical and laboratory data of a large series of patients with congenital generalized lipodystrophy. Diabetol Metab Syndr 8: 23, 2016.

9. Li H and Durbin R: Fast and accurate long-read alignment with Burrows-Wheeler transform. Bioinformatics 26: 589-595, 2010.

10. Van der Auwera GA, Carneiro MO, Hartl C, Poplin R, Del Angel G, Levy-Moonshine A, Jordan T, Shakir K, Roazen D, Thibault J, et al: From FastQ Data to High Confidence Variant Calls: The Genome Analysis Toolkit Best Practices Pipeline. Curr Protoc Bioinformatics 43: 11.10.1-11.10.33, 2013.

11. Karczewski KJ, Francioli LC, Tiao G, Cummings BB, Alföldi J, Wang Q, Collins RL, Laricchia KM, Ganna A, Birnbaum DP, et al: Variation across 141,456 human exomes and genomes reveals the spectrum of loss-of-function intolerance across human protein-coding genes. bioRxiv: 531210, 2019.

12. Shen $Y$ and Wu BL: Designing a simple multiplex ligation-dependent probe amplification (MLPA) assay for rapid detection of copy number variants in the genome. J Genet Genomics 36: 257-265, 2009.

13. Liu Y, Li D, Ding Y, Kang L, Jin Y, Song J, Li H and Yang Y: Further delineation of AGPAT2 and BSCL2 related congenital generalized lipodystrophy in young infants. Eur J Med Genet 62: 103542,2019

14. Su X, Lin R, Huang Y, Sheng H, Li X, Ting TH, Liu L and Li X: Clinical and Mutational Features of Three Chinese Children with Congenital Generalized Lipodystrophy. J Clin Res Pediatr Endocrinol 9: 52-57, 2017.

15. Richards S, Aziz N, Bale S, Bick D, Das S, Gastier-Foster J, Grody WW, Hegde M, Lyon E, Spector E, et al; ACMG Laboratory Quality Assurance Committee: Standards and guidelines for the interpretation of sequence variants: A joint consensus recommendation of the American College of Medical Genetics and Genomics and the Association for Molecular Pathology. Genet Med 17: 405-424, 2015.

16. Garg A and Misra A: Lipodystrophies: Rare disorders causing metabolic syndrome. Endocrinol Metab Clin North Am 33: 305-331, 2004.

17. Marchuk DS, Crooks K, Strande N, Kaiser-Rogers K, Milko LV, Brandt A, Arreola A, Tilley CR, Bizon C, Vora NL, et al: Increasing the diagnostic yield of exome sequencing by copy number variant analysis. PLoS One 13: e0209185, 2018. 
18. Samarakoon PS, Sorte HS, Kristiansen BE, Skodje T, Sheng Y, Tjønnfjord GE, Stadheim B, Stray-Pedersen A, Rødningen OK and Lyle R: Identification of copy number variants from exome sequence data. BMC Genomics 15: 661, 2014.

19. Tan R, Wang Y, Kleinstein SE, Liu Y, Zhu X, Guo H, Jiang Q, Allen AS and Zhu M: An evaluation of copy number variation detection tools from whole-exome sequencing data. Hum Mutat 35: 899-907, 2014.

20. Miyatake S, Koshimizu E, Fujita A, Fukai R, Imagawa E, Ohba C, Kuki I, Nukui M, Araki A, Makita Y, et al: Detecting copy-number variations in whole-exome sequencing data using the eXome Hidden Markov Model: An 'exome-first' approach. J Hum Genet 60: 175-182, 2015.

21. Yao R, Yu T, Qing Y, Wang J and Shen Y: Evaluation of copy number variant detection from panel-based next-generation sequencing data. Mol Genet Genomic Med 7: e00513, 2019.
22. Magré J, Delépine M, Khallouf E, Gedde-Dahl T Jr, Van Maldergem L, Sobel E, Papp J, Meier M, Mégarbané A, Bachy A, et al; BSCL Working Group: Identification of the gene altered in Berardinelli-Seip congenital lipodystrophy on chromosome 11q13. Nat Genet 28: 365-370, 2001.

23. Wee K, Yang W, Sugii S and Han W: Towards a mechanistic understanding of lipodystrophy and seipin functions. Biosci Rep 34: 34, 2014.

(i) (3) This work is licensed under a Creative Commons Attribution-NonCommercial-NoDerivatives 4.0 International (CC BY-NC-ND 4.0) License. 